\title{
Editorial
}

\section{Qualitative Theory of Functional Differential and Integral Equations}

\author{
Cemil Tunç, ${ }^{1}$ Mouffak Benchohra, ${ }^{2}$ Bingwen Liu, ${ }^{3}$ \\ Muhammad N. Islam, ${ }^{4}$ and Samir H. Saker ${ }^{5}$ \\ ${ }^{1}$ Department of Mathematics, Faculty of Science, Yuzuncu Yil University, 65080 Van, Turkey \\ ${ }^{2}$ Laboratoire de Mathematiques, Université Djillali Liabès de Sidi Bel Abbes, 22000 Sidi Bel Abbes, Algeria \\ ${ }^{3}$ College of Mathematics, Physics and Information Engineering, Jiaxing University, Jiaxing, Zhejiang 314001, China \\ ${ }^{4}$ Department of Mathematics, University of Dayton, Dayton, OH 45469, USA \\ ${ }^{5}$ Department of Mathematics, Damietta Faculty of Science, Mansoura University, Mansoura 35516, Egypt
}

Correspondence should be addressed to Cemil Tunç; cemtunc@yahoo.com

Received 10 November 2014; Accepted 10 November 2014

Copyright (C) 2015 Cemil Tunç et al. This is an open access article distributed under the Creative Commons Attribution License, which permits unrestricted use, distribution, and reproduction in any medium, provided the original work is properly cited.

Functional differential equations arise in many areas of science and technology: whenever a deterministic relationship involving some varying quantities and their rates of change in space and/or time (expressed as derivatives or differences) is known or postulated. This is illustrated in classical mechanics, where the motion of a body is described by its position and velocity as the time varies. In some cases, this differential equation (called an equation of motion) may be solved explicitly. In fact, differential equations play an important role in modelling virtually every physical, technical, biological, ecological, and epidemiological process, from celestial motion, to bridge design, to interactions between neurons, to interaction between species, to spread of diseases with a population, and so forth. Also many fundamental laws of chemistry can be formulated as differential equations and in economy differential equations are used to model the behavior of complex systems. However, the mathematical models can also take different forms depending on the time scale and space structure of the problem; it can be modeled by delay differential equations, difference equations, partial delay differential equations, partial delay difference equations, or the combination of these equations.

When necessary, random effects and sudden effects can also be considered in modelling problems. The mathematical theory of differential equations first developed, together with the sciences, where the equations had originated and where the results found applications. Differential and difference equations such as those used to solve real-life problems may not necessarily be directly solvable, that is, do not have closed form solutions. Only the simplest equations admit solutions given by explicit formulas; however, some properties of solutions of a given differential equation may be determined without finding their exact form. If a self-contained formula for the solution is not available, the solution may be numerically approximated using computers. In this case a recurrence relation is needed which is an equation that recursively defines a sequence: each term of the sequence is defined as a function of the preceding terms. A difference equation is a specific type of recurrence relations. Solving a recurrence relation means obtaining a closed form solution: a nonrecursive function.

However, diverse problems, sometimes originating in quite distinct scientific fields, may give rise to identical differential and difference equations. Whenever this happens, mathematical theory behind the equations can be viewed as a unifying principle behind diverse phenomena; see, for example, the books by Brauer and Castillo Chavize [1], Diekmann et al. [2], Gopalsamy [3], Gyori and Ladas [4], Kocic and Ladas [5], Kolmonovskii and Myshkis [6], Lakshmikantham et al. [7], May and Anderson [8], Murray [9], Sharkovsky et al. [10], and $\mathrm{Wu}[11]$.

A delay differential equation (DDE) is an equation for a function of a single variable, usually called time, in which the derivative of the function at a certain time is given in terms 
of the values of the function at earlier times. A functional equation (FE) is an equation involving an unknown function for different argument values. The equations $x(2 t)+2 x(3 t)=$ $1, x(t)=2 x(t+1)-[x(t-2)]^{2}$, and so forth are examples of this type. The differences between the argument values of an unknown function and $t$ in a FE are called argument deviations. If all argument deviations are constants, then FE is called a difference equation. Combining the notions of differential and functional equations, we obtain the notion of functional differential equation (FDE) or equivalently differential equations with deviating argument. Thus, this is an equation concerning the unknown function and some of its derivatives for, in general, different argument values (present, past, or future). The order of a FDE is the order of the highest derivative of the unknown function entering in the equation. So, a FE may be regarded as FDE of order zero. Hence, the notion of FDE generalizes all equations of mathematical analysis for functions of a continuous argument.

The qualitative study of functional differential equations and difference equations is a wide field in pure and applied mathematics, physics, meteorology, engineering, and population dynamics. All of these disciplines are concerned with the properties of these equations of various types. Pure mathematics focuses on the existence and uniqueness of solutions; for global existence and uniqueness theorems for differential equations, we refer to the books $[6,7,12]$ and for basic theory of difference equations, we refer to the books [13]. On the other hand, applied mathematics emphasizes the rigorous justification of the qualitative behavior of solutions (oscillation, periodic orbits, persistence, permanence, stability, global attractivity, Hopf bifurcation, Floquet theory, control, synchronization, etc.) $[2,14,15]$. On the other hand the study of integral inequalities has received a lot of attention in the literature and has become a major field in pure and applied mathematics; we refer to the recent book [16].

The oscillation theory, stability theory, bifurcation theory, existence of periodic solutions and convergence of solutions as parts of the qualitative theory of differential and difference equations have been developed rapidly in the past thirty years and some interesting books have been written in these subjects. We refer the reader to the books [17-21]. In this special issue we will consider some papers in all the above different areas and hope that the reader will find in this special issue some important results.

\section{Cemil Tunç Mouffak Benchohra Bingwen Liu Muhammad N. Islam Samir H. Saker}

[3] K. Gopalsamy, Stability and Oscillation in Delay Differential Equations of Population differentials, Kluwer Academic, Dordrecht, The Netherlands, 1992.

[4] I. Gyori and G. Ladas, Oscillation Theory of Delay Differential Equations with Applications, Clarendon Press, Oxford, UK, 1991.

[5] V. L. Kocic and G. Ladas, Global Behavior of Nonlinear Difference Equations of Higher Order with Applications, Kluwer Academic, Dordrecht, Germany, 1993.

[6] V. Kolmonovskii and A. Myshkis, Introduction to the Theory and Applications of Functional Differential Equations, Kluwer Academic, Dordrecht, Germany, 1999.

[7] V. Lakshmikantham, D. D. Bainov, and P. S. Simeonov, Theory of Impulsive Differential Equations, World Scientific, Singapore, 1989.

[8] R. May and R. M. Anderson, Infectious Diseases of Humans: Differentials and Control, Oxford Science, 1995.

[9] J. D. Murray, Mathematical Biology, Springer, New York, NY, USA, 1993.

[10] A. N. Sharkovsky, Y. L. Miastrenko, and E. Y. Romamenko, Difference Equations and Their Applications, Kluwer Academic Publishers, Dordrecht, The Netherlands, 1993.

[11] J. H. Wu, Theory and Applications of Partial Functional Differential Equations, Springer, New York, NY, USA, 1996.

[12] J. Hale, Theory of Functional Different ial Equations, Springer, New York, NY, USA, 1977.

[13] R. P. Agarwal, Difference Equations and Inequalities, Theory, Methods and Applications, Marcel Dekker, New York, NY, USA, 2nd edition, 2000.

[14] S. N. Elaydi, An Introduction to Difference Equations, Springer, New York, NY, USA, 1999.

[15] I. T. Kiguradze and T. A. Chanturia, Asy Mptotic Properties of Solutions of Nonatunomous Ordinary Differential Equations, Kluwer Academic, Dordrecht, The Netherlands, 1993.

[16] R. Agarwal, D. O’Regan, and S. H. Saker, Dynamic Inequalities on Time Scales, Springer, 2014.

[17] R. P. Agarwal, S. R. Grace, and D. O'Regan, Oscillation Theory for Second Order Dynamic Equations, vol. 5 of Series in Mathematical Analysis and Applications, Taylor \& Francis, London, UK, 2003.

[18] D. D. Bainov and D. P. Mishev, Oscillation Theory for Neutral Differential Equations with Delay, Adam Hilger, New York, NY, USA, 1991.

[19] S. H. Saker, Oscillation Theory of Delay Differential and Difference Equations: Second and Third Orders, Verlag Dr. Müller, Saarbrücken, Germany, 2010.

[20] S. H. Saker, Oscillation Theory of Dynamic Equations on Time Scales: Second and Third Orders, Lambert Academic Publishing, 2010.

[21] R. P. Agarwal, D. O’Regan, and S. H. Saker, Oscillation and Stability of Delay Models in Biology, Springer, 2014.

\section{References}

[1] F. Brauer and C. Castillo Chavize, Mathematical Models in Population Biology and Epidemiology, Springer, 2001.

[2] O. Diekmann, S. A. van Gils, S. M. V. Lunel, and H.-O. Walther, Delay Equations, Functional, Complex and Nonlinear Analysis, Springer, New York, NY, USA, 1995. 


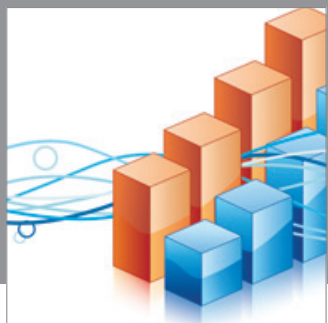

Advances in

Operations Research

mansans

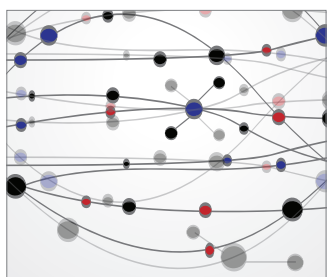

The Scientific World Journal
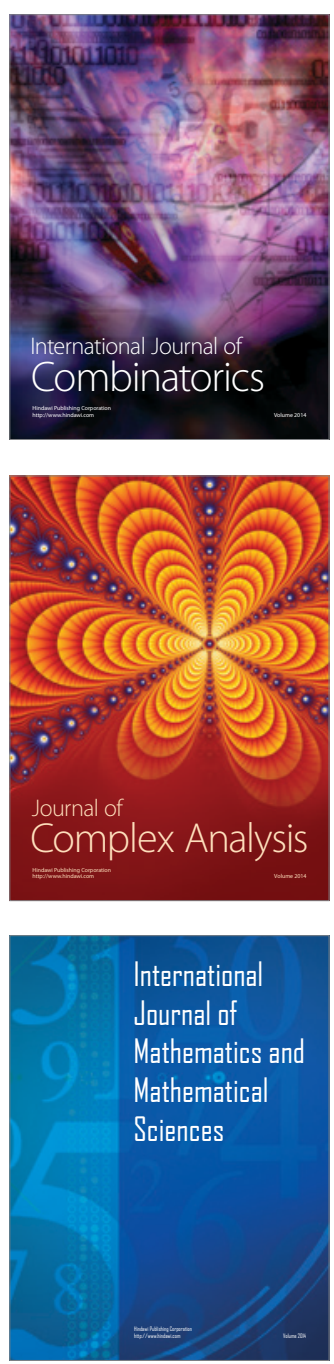
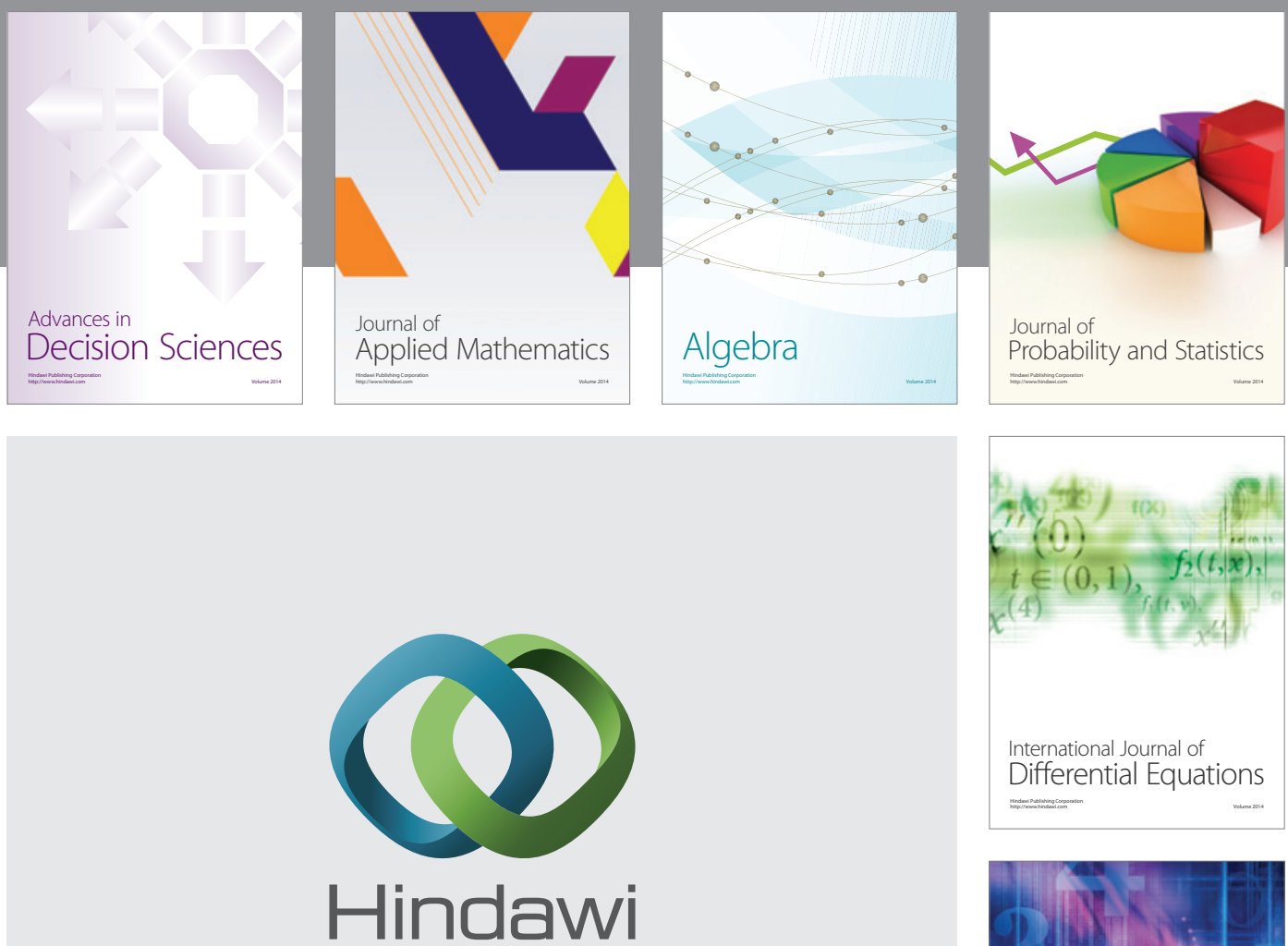

Submit your manuscripts at http://www.hindawi.com
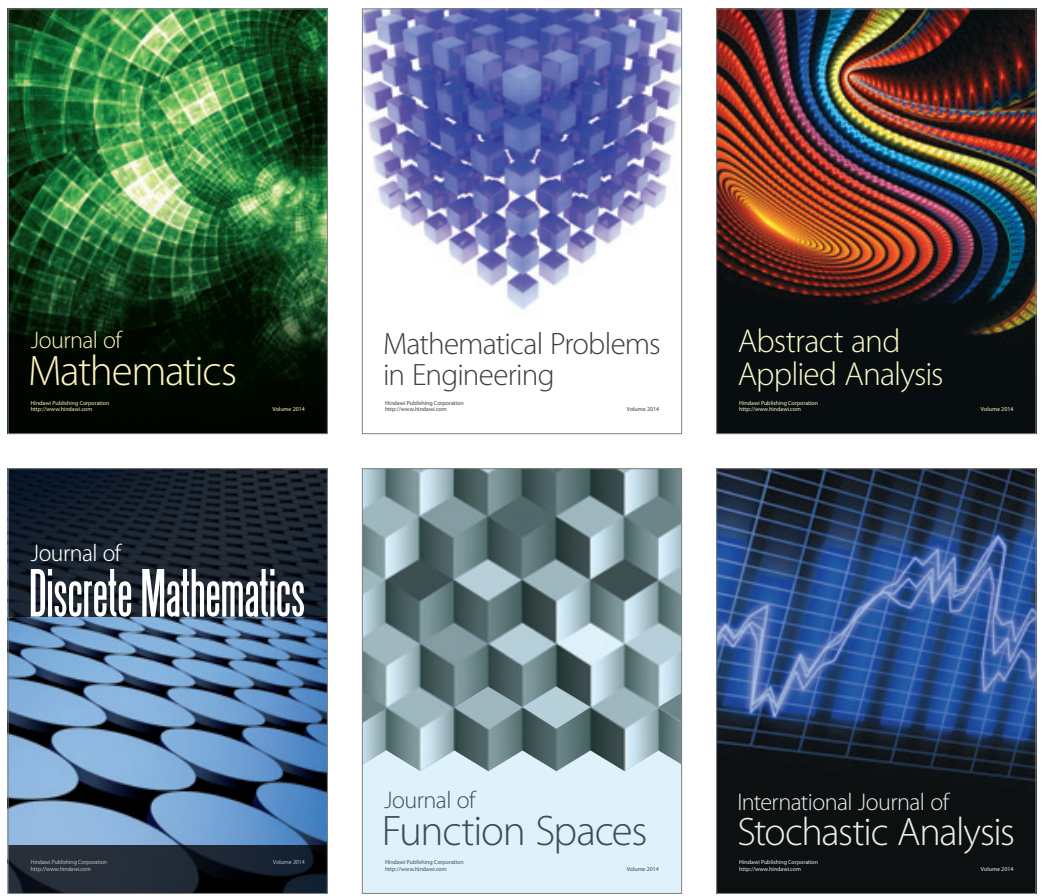

Journal of

Function Spaces

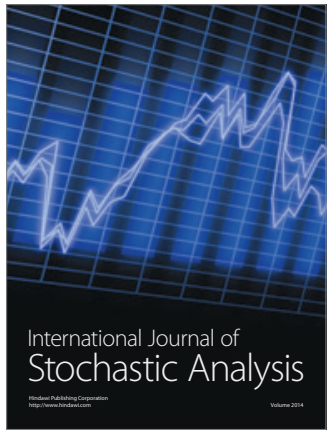

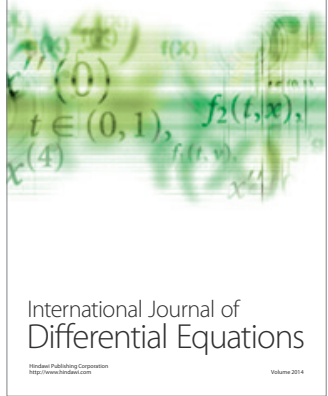
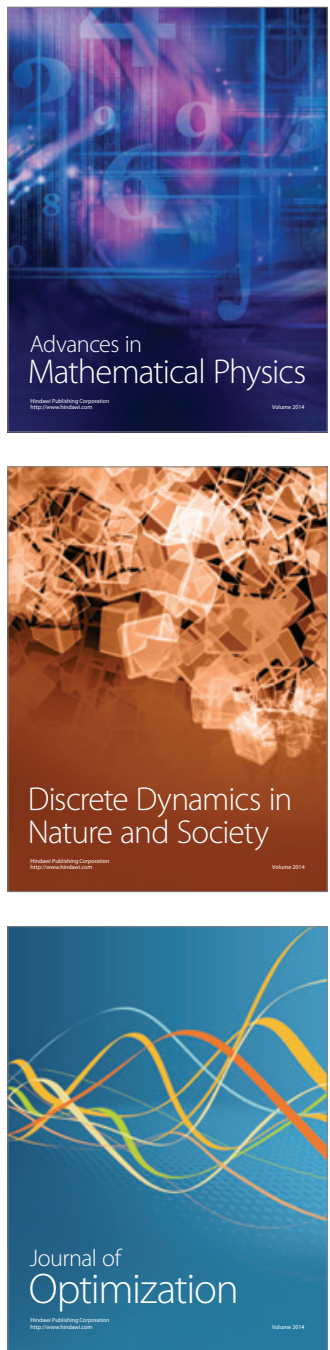